OPEN $\%$ ACCESS Freely available online

http://www.banglajol.info/index.php/BJID/index

Review Article

Bangladesh Journal of Infectious Diseases

June 2018, Volume 5, Number 1

ISSN (Online) 2411-670X; ISSN (Print) 2411-4820

DOI: http://dx.doi.org/10.3329/bjid.v5i1.37714

\title{
Occult Hepatitis B Virus Infection: A Review Update
}

\author{
Arifa Akram \\ Medical Officer, Department of Virology, Institute of Epidemiology Disease control and research, Dhaka, Bangladesh
}

[Received: 17 April 2018; Accepted: 12 May 2018; Published: 1 June 2018]

\begin{abstract}
Occult HBV infection (OBI) is defined as HBV DNA detection in serum or in the liver by sensitive diagnostic tests in HbsAg negative individuals with or without serologic markers of previous viral exposure. Since OBI was first described in the late 1970s, there has been increasing concern in this topic. OBI can be both a source of virus contamination in blood and organ donations and the reservoir for full blown hepatitis after reactivation. HBV reactivation depends on viral and host factors but these associations have not been analyzed thoroughly. Although the exact mechanism of OBI yet not proved, intrahepatic persistence of viral covalently closed circular DNA under the host's strong immune suppression of HBV replication and gene expression seems to be a cause. Current evidence suggests that OBI can favour the progression of fibrosis, cirrhosis, hepatocellular carcinoma and post transfusion hepatitis (PTH). Epidemiological data regarding the global prevalence of OBI vary due to the use of detection methods of different sensitivity and specificity. Appropriate diagnostic techniques must be adopted. Sensitive HBV DNA amplification assay is the gold standard assay for detection of OBI. [Bangladesh Journal of Infectious Diseases 2018;5(1):32-38]

Keywords: Hepatitis B virus; Hepatitis B virus DNA; Occult hepatitis B virus infection; Chronic liver disease; Hepatocellular carcinoma; Hepatitis B surface antigen; Anti-HBc screening

Correspondence: Dr. Arifa Akram, Medical Officer, Department of Virology, Institute of Epidemiology Disease control and research, Dhaka, Bangladesh; Cell no.: +8801816296249; Email: drbarna43@gmail.com

Conflict of interest: There is no conflict of interest to any of the authors of this article.

Funding agency: The study was not funded by any authority.

Contribution to authors: Akram had searched the literature, had prepared the manuscript and had revised it properly

How to cite this article: Akram A. Occult Hepatitis B Virus Infection: A Review Update. Bangladesh J Infect Dis 2018;5(1):3238

Copyright: (92018. Akram. Published by Bangladesh Journal of Infectious Diseases. This article is published under the Creative Commons CC BY-NC License (https://creativecommons.org/licenses/by-nc/4.0/). This license permits use, distribution and reproduction in any medium, provided the original work is properly cited, and is not used for commercial purposes.
\end{abstract}

\subsection{Introduction}

Hepatitis B virus (HBV) infection is an important global public issue. According to European Association for the study of the liver (EASL), about one third of the world's population have serological evidence of past or present hepatitis B virus (HBV) infection, and more than 350 million people may be affected by chronic HBV infection. In addition, chronic HBV infection is the worldwide primary cause of cirrhosis and hepatic cellular carcinoma, and it is among the top ten causes of death. The clinical progression of $\mathrm{HBV}$ is variable, ranging from mild liver disease to fulminate hepatitis, cirrhosis, or hepatic cellular carcinoma (HCC). In some individuals, in whom the HBV infection 
persists, serological markers can identify different clinical states of viral persistence ${ }^{1}$.

Implementation of hepatitis B surface antigen (HBsAg) in routine screening of blood donors in the early 1970s has greatly enhanced transfusion safety. The incidence of transfusion-transmitted hepatitis B has been steadily reduced over the last four decades $^{2}$. However, it was demonstrated that HBV transmission by blood components negative for HBsAg can still occur ${ }^{3}$ and HBV transmission remains the most frequent transfusion-transmitted viral infection ${ }^{4}$; thus, the term occult hepatitis B virus infection (OBI) was introduced. Almost 30 years ago OBI was reported for the first time in a case report of HBV infection through blood transfusion by an antibody to hepatitis B core antigen (anti-HBc) only positive donor ${ }^{5}$.

In 2008, the European Association for the study of the liver (EASL) defined occult hepatitis B virus infection (OBI) as the "presence of hepatitis B virus (HBV) DNA in the liver (with detectable or undetectable HBV DNA in the serum) of individuals testing hepatitis $\mathrm{B}$ surface antigen (HBsAg) negative by currently available assays" ${ }^{1}$.

Allain reported OBI in several clinical contexts including: (1) recovery from past infection indicated by the presence of hepatitis $B$ surface antibody (anti-HBs); (2) chronic hepatitis with surface gene escape mutants that are not recognized by current assays; (3) chronic carriage without any marker of HBV infection other than HBV DNA (referred to as "seronegative"); and (4) most commonly in endemic areas, chronic carriage stage with HBsAg too low to be detected and recognized by the presence of anti-HBc as the only serological marker (referred to as "anti-HBc alone" or "isolated anti-HBc") ${ }^{6}$.

\subsection{Serological Pattern of Occult Hepatitis B Infection}

OBI can be classified into 2 groups, seropositive OBI [anti-HBc and/or anti-hepatitis B surface (antiHBs) positive] and seronegative OBI (anti-HBc and antiHBs negative), on the basis of the HBV antibody profile.

\subsection{Seropositive Occult HBV infection (OBI)}

These are OBI subjects with anti-HBc and/or positive anti-HBs in which serum $\mathrm{HBsAg}$ is not detected because of the resolution of acute hepatitis B (after a few months of HBsAg carriage) or after years of chronic HBsAg positive infection ${ }^{7}$. Thirty- five percent of patients with OBI have positive antiHBs and forty-two percent of have positive anti$\mathrm{HBc}^{8}$. The HBV DNA detection rate is higher in individuals who are positive anti-HBc but negative for anti-HBs. When patients give a positive result for both antibodies, they have intermediate HBV DNA levels. One explanation for this serological pattern is that positive anti-HBc patients with chronic HBV infection clear HBsAg to an undetectable level, with or without anti-HBs: this pattern is associated with older age and anti-HBe ${ }^{1}$.

\subsection{Seronegative Occult HBV infection (OBI)}

Patients who are not positive for anti-HBc and antiHBs represent twenty-two percent of OBI patients ${ }^{8}$. They have very low levels of HBV DNA ${ }^{9}$. This pattern of antibodies may appear from the beginning of the infection when patients have not yet developed positive hepatitis B specific antibody ("primary OBI") or because of clearance of the hepatitis B specific antibodies ${ }^{7}$. Therefore, this pattern should always be kept in mind, because almost anybody can be a potential carrier of occult $\mathrm{B}$ hepatitis ${ }^{1}$. Moreover, there are cases termed as "False" OBI. They are carriers of mutations in HBsAg (in the $S$ gene) that are not recognized by some routine detection assays. In these cases, the DNA result resembles other cases of HBV, because they are usually positive for $\mathrm{HBsAg}^{7}$.

\subsection{Epidemiology}

The prevalence of OBI is reported to range from $1 \%$ to $95 \%$ worldwide. These prevalence rates are influenced by several factors as follows: (1) geographic differences (endemicity); (2) different patient characteristics, including the presence of co morbid diseases such as chronic hepatitis $\mathrm{C}$; and (3) and the different diagnostic techniques used, which have different sensitivity ${ }^{[7,10,11]}$. OBI was reported at a higher rate in an HBV endemic area such as East Asia where $41 \%-90 \%$ of the population had prior exposure to $\mathrm{HBV}$, and less frequently in the low endemic areas such as North America, where only 5\%-20\% of the population had previous exposure. Several studies on blood donors with similar cohorts have reported a $0.1 \%-1.05 \%$ prevalence of $\mathrm{OBI}$ in $\mathrm{HBs} \mathrm{Ag}$-negative, anti-HBc-positive donors from North America, 0\%-1.59\% in donors from Europe, and up to $6 \%$ in donors from an endemic $\operatorname{area}^{12}$.

However, OBI prevalence seems to be higher among subjects at high risk for HBV infection and with liver disease than among individuals at low risk of infection and without liver disease ${ }^{13}$. The 
prevalence of OBI is estimated to be $4-25 \%$ in anti $\mathrm{HBc}$-positive patients ${ }^{14}$. OBI is more prevalent in certain groups such as HCV and HIV populations. Many studies have found a higher OBI prevalence in subjects with than those without chronic liver disease. There is a wide variation in prevalence of OBI in different case series in patients with cryptogenic chronic hepatitis, HCC, and in HCV-or HIV-infected individuals. This variability depends on the difference in endemicity of HBV infection, utilized assays in the studies, and studied populations in different parts of the world ${ }^{15}$.

The prevalence of OBI also differs according to the sensitivity of HBV DNA or HBsAg testing. There are various amplification methods for detecting HBV DNA, and the HBV genome target sites are also different. Some commercial assays are more sensitive than others at detecting HBsAg mutants. The type of sample used (liver or serum) or number of samplings can also have some effect on the diagnosis of OBI. Indeed, as serum HBV DNA levels seem to fluctuate in OBI, serial sample is more useful to identify $\mathrm{OBI}^{12}$.

\subsection{Molecular Mechanisms Associated with OBI 4.1. Mutations and Deletions in the HBV Genome}

Sequence variation in HBV genomes, including (i) mutations in the "a" determinant of HBsAg, (ii) treatment-associated mutations, (iii) splicing, and (iv)mutations in the pre-S region have been linked to occult $\mathrm{HBV}$ infection.

Mutations in the "a" determinant of HBsAg. A mutation in the "a" determinant of the surface antigen was one of the earliest recognized mechanisms leading to occult $\mathrm{HBV}$ infection. Mutations in HBsAg lead to conformational changes rendering the protein undetectable by some of the commercially available HBsAg assays ${ }^{16}$. The inability of some but not all commercial assays to detect HBsAg from samples associated with mutations in the "a" determinant is well documented. The emergence of " $a$ " determinant mutants is a serious health concern not only because they are not detectable by some commercial HBsAg assays but also because they can infect both unvaccinated and vaccinated individuals ${ }^{17}$.

Also, a series of mutations caused by treatment with lamivudine have been described, which are used to combat viral infections. This drug causes changes in the polymerase and surface genes. The mutations Q563S, V539I, M204I, and L180M/M204I confer resistance to lamivudine therapy and reduce the affinity of the polymerase to its natural substrates, the dNTPs, leading to lower viral replication. Even a reduction in the binding antibody to a range of $S$ mutants derived from commonly selected lamivudine-resistant HBV mutants has been shown. The expressed proteins containing these mutations had an altered antigenicity and may have the potential to escape neutralization by anti-HBs antibody ${ }^{18}$.

Another mutation in the surface gene (G458A) can alter mRNA splicing of the gene, affecting exportation of mRNA and DNA folding, leading to zero expression of HBsAg ${ }^{19}$. The participation of the $S$ gene is important to the viral infectivity of $\mathrm{HBV}$, since it is known that high levels of the S gene promote the assembly and secretion of excessive amounts of noninfectious HBsAg particles from the cell, independently of the release of the virion. Alteration of splicing by mutations in the genome may also interfere with viral replication via the pgRNA. Splicing of the pgRNA produces a non-functional viral DNA of reduced size (from 3100-2000 nucleotides) that can, however, encapsidate and export to the medium ${ }^{20}$.

In addition, an alternative RNA splicing event has been reported (reduction of 2986 nucleotides to 202) that suppresses expression of the surface protein gene without affecting the polymerase and functions related to the core or $\mathrm{X}$ proteins. This splicing generates intracellular virus particles, without the surface protein, that subsequently accumulate mutations due to the relaxation of restrictions on encryption. Such viruses are deficient in terms of autonomous propagation and cannot leave the host cell until it is lysed ${ }^{21}$.

Many reports have found mutations and rearrangements in the donor splice sites of the pre$\mathrm{S} 1$, Pres-S2/ and S genes in patients with OBI. These mutations may be associated with reduced expression and secretion of HBsAg and could affect pre-S2/S mRNA splicing. Other mutations found in the overlap region of the core gene promoter, influence the low replicative potential while variants in the Pre-Core/Core sequences reduce the efficiency of replication through the functional structure of the epsilon signal, which is essential for pre genomic encapsidation and initiation of $\mathrm{HBV}$ DNA synthesis ${ }^{18}$.

\subsection{Genome Integration of HBV}

Although the specific mechanisms that cause OBI are still not fully understood, several studies have 
suggested the possible origins of this condition. The stability and long-term persistence of viral cccDNA molecules, together with the long half-life of hepatocytes, imply that HBV infection, once it has occurred, may possibly continue for life ${ }^{22}$.

Disruption and rearrangement of genes during integration into chromosomal DNA can result in (i) loss of HBsAg in the serum, (ii) reduction in virion production, and (iii) loss of detectable HBV DNA in serum. Therefore, integration of HBV DNA represents a key mechanism underlying occult HBV infection, especially following several years of chronic HBV infection. Integrated HBV DNA rather than episomal $\mathrm{HBV}$ is frequently detected in $\mathrm{HBV}$-related $\mathrm{HCC}^{17}$.

\subsection{Epigenetic Changes}

Methylation: In the human genome, regions rich in $\mathrm{CpG}$ dinucleotides are referred to as $\mathrm{CpG}$ islands. Methylation of cytosines in $\mathrm{CpG}$ dinucleotides within $\mathrm{CpG}$ islands in gene promoters leads to gene silencing ${ }^{23}$. Methylation is a key mechanism for regulation of transcriptional activity. Methylation of HBV DNA represents a novel epigenetic mechanism that impairs HBV proteins, HBV replication, and $\mathrm{HBV}$ virion production, leading to occult HBV infection ${ }^{17}$.

Acetylation: The acetylation of histones bound to the DNA is another mechanism that regulates the transcriptional activity of HBV. Hyper acetylation of histones bound to cccDNA has been associated with increased viral replication in cell culture. A mutant $\mathrm{HBx}$ of only one nucleotide has been associated with a rapid hypo acetylation of histones bound to cccDNA, thereby weakening the recruitment of the p300 transcriptional activator ${ }^{18}$.

\subsection{Co infection with Other Microbe}

Co-infection with HCV and HBV is another factor involved in the genesis of OBI. It has been shown that the rate of clearance of $\mathrm{HBsAg}$ is 2.5 times higher in $\mathrm{HCV} / \mathrm{HBsAg}$ positive cases than in $\mathrm{HCV}$ infection only, suggesting that $\mathrm{HCV}$ is the most important hepatotropic virus in terms of enhancing the clearance of HBsAg in chronic hepatitis $\mathrm{B}^{24}$.

This has been clearly observed in recipients of blood transfusions infected with $\mathrm{HCV}$ and $\mathrm{HBV}$, where the initial appearance of HBsAg is often delayed and subsequently followed by an interval of detection of HBsAg and a reduction in the peak levels of HBV DNA ${ }^{18}$.

\subsection{Other Mechanisms}

As stated previously, several mechanisms have been proposed that, either alone or in union, participate in the development of OBI. This can be through alterations to the infectious agent itself (genomic mutations), through competition with other hepatotropic agents or through the host response to infection. This ultimately leads to zero detection of HBsAg in the serum of patients when tested with the currently available assays ${ }^{18}$.

\subsection{Methods for Diagnosis of Occult HBV Infection}

The gold standard for OBI diagnosis is the detection of HBV DNA in the DNA extraction from the liver, as cccDNA persists in the hepatocytes and HBV DNA is sometimes detected in the liver in the absence of HBV DNA in the serum. However, obtaining liver tissue is an invasive procedure; therefore, obtaining hepatic HBV DNA is difficult in clinical practice. In addition, real-time PCR based assays for serum (or plasma) HBV DNA detection have been used with sufficient sensitivity to detect OBI in many cases; hence, serum HBV DNA assays are widely used to diagnose $\mathrm{OBI}^{7}$.

DNA extracts should be amplified by highly sensitive nested-PCR, real-time PCR, and transcription based mediated amplification (TMA) technique that can detect fewer than 10 copies of HBV DNA using the oligonucleotide primers specific for different HBV genomic regions and complementary to highly conserved nucleotide sequences. Appropriate negative and positive controls should be included in each PCR experiment. In addition, periodic testing for $\mathrm{HBV}$ DNA will improve diagnosis of OBI especially in high-risk patients, as intermittent viremia can occur in occult HBV infection ${ }^{12}$

When highly sensitive HBV DNA testing cannot be performed, anti-HBc could be used as a possible surrogate marker for identifying potential seropositive OBI in cases of blood and organ donation or those receiving immunosuppressive therapy. In this case, seronegative OBI or falsenegative anti-HBc in an immunocompromised host should also be considered ${ }^{25}$. In such cases, not all anti-HBc positive individuals are positive for $\mathrm{HBV}$ DNA, and anti-HBc tests might provide falsepositive results ${ }^{7}$. Furthermore, the absence of this antibody does not exclude OBI (seronegative OBI). If this marker is used in combination with $\mathrm{HBV}$ DNA, the prevalence of $\mathrm{HBV}$ infection in the area 
should be considered, because when prevalence of anti-HBc is higher than a $50 \%$ of the donor population, a positive result is unhelpful ${ }^{1}$.

\subsection{Clinical Significance}

6.1. Transfusion: Although the risk of $\mathrm{HBV}$ transmission through blood transfusion has decreased owing to the development of sensitive and specific diagnostic assays, transfusional transmission of HBV still occurs Transmission of HBV by transfusion occurs in 3 situations: (1) blood from a donor with OBI; (2) blood from patients in the infectious window period of HBV infection; or (3) blood from a donor infected with S-escape mutant HBV infection not detected by the routinely used diagnostic HBsAg assay. The prevalence of OBI in blood donors is variable depending on the geographic area, and is higher in HBV endemic areas ${ }^{26}$.

It is generally admitted that pre-sero conversion WP infections are most likely to transmit HBV but transmission from occult $\mathrm{HBV}$ infection remains a debated subject. Occult $\mathrm{HBV}$ is transmissible through blood transfusion in $\mathrm{HBV}$-naive recipients. Post-transfusion hepatitis B virus (HBV) infection still occurs, although its incidence has been found to be substantially reduced since the introduction of screening for HBsAg in blood donors. A similar study was recently conducted in India and showed that a considerable number of HBV-infected donors remain undetected, if only $\mathrm{HBsAg}$ is used for screening. A recent study conducted in Taiwan showed that in HBV hyperendemic areas, occult hepatitis B transfusion might not lead to HBsAg carriage or post-transfusion hepatitis. OBI blood donors have very low HBV replication, and normal liver biochemistry and histology, conferring a favorable prognosis ${ }^{5}$.

Nucleic acid testing (NAT) for donor screening detects $\mathrm{HBV}$ infection in the window period (before the appearance of $\mathrm{HBsAg}$ ) as well as OBI, indicating the presence of HBV DNA in the absence of HBsAg. Therefore, the introduction of NAT has further decreased the risk of HBV transmission through blood transfusion. However, cost effectiveness and availability of NAT should be considered before clinical application. Where HBV DNA testing is not available, such as in developing countries, testing for anti-HBc is strongly recommended ${ }^{[7,25]}$.

\subsection{Organ transplantation: $\mathrm{OBI}$ in a} transplantation donor is important because there is a risk of $\mathrm{HBV}$ transmission from an $\mathrm{OBI}$-seropositive donor, and severe $\mathrm{HBV}$ reactivation can occur in some of these cases during immunosuppression. As the hepatocytes are the reservoir of HBV cccDNA, the rate of transmission is higher in orthotopic liver transplantation compared to other organ transplantations such as kidney, bone marrow, and heart ${ }^{12}$. With regard to the management of these patients, it is not known if prior hepatitis B immunization with an optimal anti-HBs response can modulate or abort the infection. Prophylaxis with antiviral agents prevents reactivation of $\mathrm{OBI}$ in most of these cases ${ }^{27}$.

6.3. Hemodialysis: Hemodialysis patients are at increased risk of parenterally transmitted infections because they are in an immunosuppressed state and exposed to invasive procedures, share the same dialysis machine, and receive more transfusions than the general population. The relatively low acceptance and response rates to the HBV vaccine among dialysis patients also likely contributes to OBI transmission in hemodialysis patients. The prevalence of OBI in hemodialysis patients varies from $0 \%$ to $54 \%$ according to the diagnostic techniques or HBV endemicity, and several studies suggest that OBI could be a source of viral spread both to other patients and staff within the hemodialysis units. Therefore, patients and staff need HBV vaccine boosts to maintain levels of protective antibody to $\mathrm{HBsAg}$ (anti-HBs) ${ }^{28}$.

6.4. OBI and CLD: OBI has been detected in patients with cryptogenic chronic liver disease ${ }^{29}$ and could be associated with progression of liver fibrosis and cirrhosis development in these patients. HBV-infected patients might present with progressive reduction of viral replication and serum HBsAg levels. HBsAg might disappear over time, despite the presence of severe liver injury that has been provoked by overt hepatitis $\mathrm{B}$, and then maintained once the occult HBV status has been established $^{27}$. It has been reported that close monitoring of serum HBV-DNA levels and liverenzyme levels could be useful in the management of patients with OBI and cryptogenic liver disease in two respects: (1) to predict the risk of cirrhosis or HCC; and (2) to decide on the possibility of antiviral treatment to prevent HBV reactivation or transmission in the case of transplantation. However, the role of OBI in accelerating the development of cirrhosis is still unresolved. Prospective studies using well defined selection criteria of patients and standardized laboratory techniques are needed ${ }^{27}$. A meta-analysis of both OBI mono-infection and co-infection with $\mathrm{HCV}$ showed that OBI is associated with chronic liver disease, with an overall 8.9-fold increased risk compared to individuals without $\mathrm{OBI}^{30}$.

6.5. OBI and HCC: OBI is supposed to be an important risk factor for $\mathrm{HCC}$ development since it 
maintains the pro-oncogenic properties typical of the overt infection. It has been suggested that the occult viral strains, maintaining the transcriptional activity and the pro-oncogenetic assets of the clear $\mathrm{HBV}$ infection (HBsAg+), may harbor a potential risk for liver cancer development ${ }^{5}$. This prooncogenic role is not only the consequence of the integration of viral DNA into the host genome. Other factors might contribute: (1) persistence of replicating virus might induce mild liver necroinflammation that continues for life; (2) occult strains usually persist as free genomes, and maintain the capacity to transcribe and replicate; and (3) OBI might contribute to progression towards cirrhosis, which is the most important risk factor for HCC development. However, further molecular pathogenesis studies and prospective molecular epidemiological studies are needed to reach the conclusion that OBI plays a major role in hepatocellular transformation. Until then, it is premature to recommend testing all $\mathrm{HBsAg}$ negative patients with $\mathrm{HCC}$ for $\mathrm{OBI}^{27}$. A study conducted in Japan confirmed the existence of serum HBV DNA in OBI as a predictor of a high hepatocellular carcinogenesis rate in a cohort of patients with non-B, non-C cirrhosis ${ }^{5}$.

\subsection{Preventive Measure}

At least one-third of patients die from $\mathrm{HBV}$ reactivation despite treatment with lamivudine. It would probably be best to administer preventive treatment for $\mathrm{HBV}$ reactivation as this would inhibit the development of hepatitis and mortality with the aim of inhibiting the replication of HBV. Current data are insufficient to recommend routine prophylaxis and antiviral therapy to prevent HBV reactivation for $\mathrm{HBV}$ DNA and $\mathrm{HBsAg}$-negative butanti-HBc and/or antiHBs positive patients, except in intense chemotherapy like rituximab. Thus early identification of virologic reactivation is essential to start antiviral therapy and prevent the occurrence of hepatitis ${ }^{31}$. Serial HBV-DNA monitoring (monthly during and after chemotherapy for at least 1 year) is a reasonable strategy recommended by the latest Japanese guidelines; in this regard multicenter clinical trial in Japanis now continued. With HBV-DNA NAT antiviral therapy begins when the result is $>30 \mathrm{IU} / \mathrm{mL}$ and with a highly sensitive HBsAg assay (low limit of detection $<0.1 \mathrm{ng} / \mathrm{mL}$ ) antiviral therapy begin when the test becomes positive. Although in blood transfusion the risk of transmission is insignificant when anti-HBs is present in the blood, caution is recommended when immune deficient patients receive anti- $\mathrm{HBc}$ positive and anti-HBs-positive donations. The use of HBV-DNA NAT and multivalent anti-HBs antibodies in the $\mathrm{HBsAg}$ assays is recommended for detection of true and false OBI, respectively, and to minimize the risk of HBV transmission through transfusion. This is important because almost $50 \%$ of transfused blood in Western Europe is given to immune deficient patients ${ }^{15}$.

\subsection{Conclusions}

OBI has recently gained increasing attention. Although the exact mechanism of OBI is unknown, OBI can be transmitted, cause reactivation of HBV, and contribute to the development of progressive liver disease and Liver cancer. Nucleic acid testing (NAT) should be implemented for detection of OBI-HBV DNA even if anti-HBc and anti-HBs were negative especially in endemic area and in suspected high-risk cases with probable previous exposure before blood and organ donation, transplantation, and chemotherapy and in hemodialysis and cryptogenic chronic hepatitis. So, physicians should focus on the appropriate management of these patients, and further studies to clarify the clinical significance of OBI are needed.

\section{References}

1. Ocana S, Casas ML, Buhigas I, Lledo JL. Diagnostic strategy for occult hepatitis B virus infection. World $\mathrm{J}$ Gastroenterol 2011;17(12):1553-1557

2. Liu Y, Li P, Li C, Zhou J, Wu C, Zhou YH. Detection of hepatitis B virus DNA among accepted blood donors in Nanjing, China. Virol J 2010;7:193

3. Liu CJ, Chen DS, Chen PJ. Epidemiology of HBV infection in Asian blood donors: emphasis on occult HBV infection and the role of NAT. J Clin Virol 2006; 36:S33-44

4. Niederhauser C, Mansouri Taleghani B, Graziani M, Stolz M, Tinguely C, Schneider P. Blood donor screening: how to decrease the risk of transfusion-transmitted hepatitis B virus? Swiss Med Wkly 2008;138:134-141

5. Said ZNA. An overview of occult hepatitis B virus infection. World J Gastroenterol 2011; 17(15): 1927-1938

6. Allain JP. Occult hepatitis B virus infection. Transfus Clin Biol 2004; 11: 18-25

7. Raimondo G, Allain JP, Brunetto MR, Buendia MA, Chen DS, Colombo M, et al. Statements from the Taormina expert meeting on occult hepatitis B virus infection. J Hepatol 2008; 49: 652-657

8. Torbenson M, Thomas DL. Occult hepatitis B. Lancet Infect Dis 2002; 2: 479-486

9. Hollinger FB, Sood G. Occult hepatitis B virus infection: a covert operation. J Viral Hepat 2010; 17: 1-15

10. Schmeltzer P, Sherman KE. Occult hepatitis B: clinical implications and treatment decisions. Dig Dis Sci 2010; 55: 3328-3335

11. Samal J, Kandpal M, Vivekanandan P. Molecular mechanisms underlying occult hepatitis B virus infection. Clin Microbiol Rev 2012; 25: 142-163

12. Kwak MS, Kim YJ. Occult hepatitis B virus infection. World J Hepatol 2014; 6(12): 860-869

13. Raimondo G, Navarra G, Mondello S, Costantino L, Colloredo G, Cucinotta E, Di Vita G, Scisca C, Squadrito G, 
Pollicino T. Occult hepatitis B virus in liver tissue of individuals without hepatic disease. J of hepatol. 2008; 48(5):743-746

14. Liu CJ, Chen DS, Chen PJ. Epidemiology of HBV infection in Asian blood donors: emphasis on occult HBV infection and the role of NAT. 2006 May 1;36:S33-44. J clin virol, 2006; 36: S33-S44

15. Zobeiri M. Occult hepatitis B: clinical viewpoint and management. Hepat res treat. 2013;2013

16. Raimondo G, Allain JP, Brunetto MR, Buendia MA, Chen DS, Colombo M, et al. Statements from the Taormina expert meeting on occult hepatitis $\mathrm{B}$ virus infection. $\mathrm{J}$ of hepatol. 2008; 49(4):652-7

17. Samal J, Kandpal M, Vivekanandan P. Molecular mechanisms underlying occult hepatitis B virus infection. Clin microbiol reviews 2012; 25(1):142-63

18. Morales-Romero J, Vargas G, García-Román R. Occult HBV infection: a faceless enemy in liver cancer development. Viruses 2014; 6(4):1590-611

19. Hass M, Hannoun C, Kalinina T, Sommer G, Manegold C, Günther S. Functional analysis of hepatitis B virus reactivating in hepatitis B surface antigen-negative individuals. Hepatology 2005; 42(1):93-103

20. Günther S, Sommer G, Iwanska A, Will H. Heterogeneity and common features of defective hepatitis B virus genomes derived from spliced pregenomic RNA. Virology 1997;2 38(2): 363-71

21. Van Hemert FJ, Zaaijer HL, Berkhout B, Lukashov VV. Occult hepatitis B infection: an evolutionary scenario. Virol J. 2008; 5(1): 146

22. Aller de la Fuente R, Gutiérrez ML, Garcia-Samaniego J, Fernández-Rodriguez C, Lledó JL, Castellano G. Pathogenesis of occult chronic hepatitis B virus infection. World J Gastroenterol 2011; 17(12): 1543-1548
23. Portela A, Esteller M. 2010. Epigenetic modifications and human disease. Nat. Biotechnol. 28:1057-1068

24. Sheen, I.S.; Liaw, Y.F.; Lin, D.Y.; Chu, C.M. Role of hepatitis $\mathrm{C}$ and delta viruses in the termination of chronic hepatitis B surface antigen carrier state: A multivariate analysis in a longitudinal follow-up study. J. Infect. Dis. 1994; 170 (2): 358-361

25. Raimondo G, Pollicino T, Romanò L, Zanetti AR. A 2010 update on occult hepatitis B infection. Pathol Biol 2010; 58 (4): 254-257

26. Liu CJ, Lo SC, Kao JH, Tseng PT, Lai MY, Ni YH, et al. Transmission of occult hepatitis B virus by transfusion to adult and pediatric recipients in Taiwan. J Hepatol 2006; 44: 39-46 27. Lledó JL, Fernández C, Gutiérrez ML, Ocaña S. Management of occult hepatitis B virus infection: An update for the clinician. World J Gastroenterol 2011; 17(12): 15631568

28. Motta JS, Mello FC, Lago BV, Perez RM, Gomes SA, Figueiredo FF. Occult hepatitis B virus infection and lamivudineresistant mutations in isolates from renal patients undergoing hemodialysis. J Gastroenterol Hepatol 2010; 25: 101-106

29. Fang Y, Shang QL, Liu JY, Li D, Xu WZ, Teng X, et al. Prevalence of occult hepatitis B virus infection among hepatopathy patients and healthy people in China. J Infect 2009; 58: 383-388

30. Covolo L, Pollicino T, Raimondo G, Donato F. Occult hepatitis B virus and the risk for chronic liver disease: a metaanalysis. Dig Liver Dis 2013; 45: 238-244

31. Manzano-Alonso ML, Castellano-Tortajada G. Reactivation of hepatitis B virus infection after cytotoxic chemotherapy or immunosuppressive therapy. World J Gastroenterol 2011;17(12):1531 